
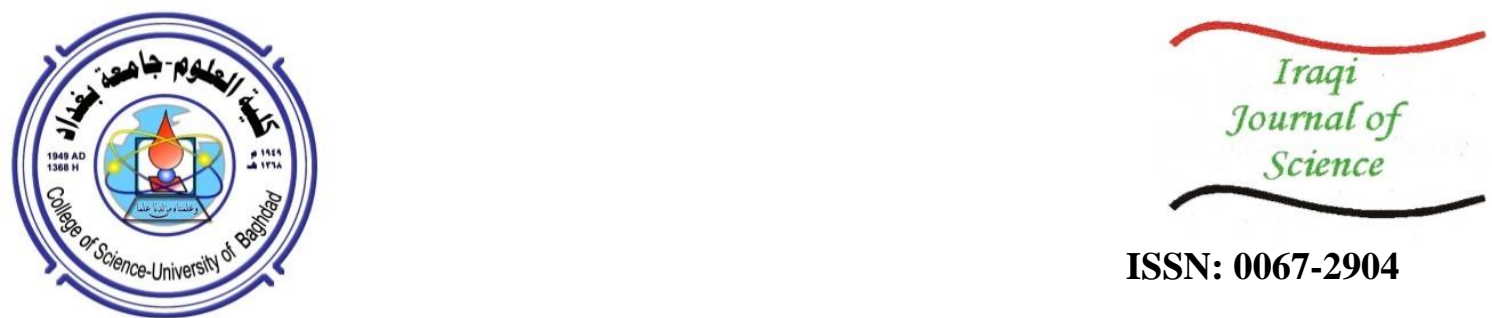

ISSN: 0067-2904

\title{
A Reliable Iterative Transform Method for Solving an Epidemic Model
}

\author{
Reem Waleed Huisen*, Sinan H. Abd Almjeed, Areej Salah Mohammed \\ Department of mathematics, College of Education for pure Sciences/Ibn-AL-Haithem, University of Baghdad, \\ Baghdad, Iraq
}

Received: $21 / 12 / 2020$

Accepted: 27/2/2021

\begin{abstract}
The main purpose of the work is to apply a new method, so-called LTAM, which couples the Tamimi and Ansari iterative method (TAM) with the Laplace transform (LT). This method involves solving a problem of non-fatal disease spread in a society that is assumed to have a fixed size during the epidemic period. We apply the method to give an approximate analytic solution to the nonlinear system of the intended model. Moreover, the absolute error resulting from the numerical solutions and the ten iterations of LTAM approximations of the epidemic model, along with the maximum error remainder, were calculated by using MATHEMATICA ${ }^{\circledR} 11.3$ program to illustrate the effectiveness of the method.
\end{abstract}

Keywords: Iterative method, Laplace transform, epidemic model, approximate solution.

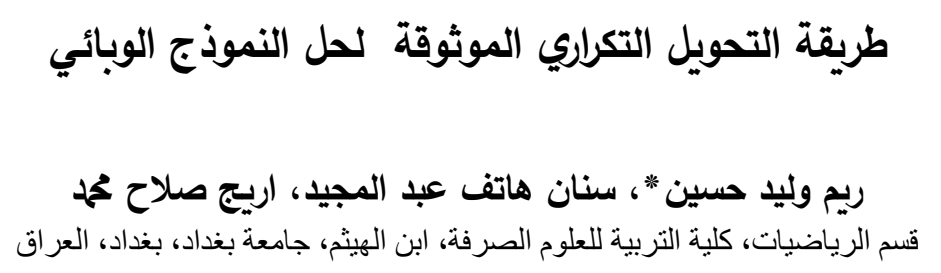

\section{Introduction}

The problem of the spread of non-fatal infectious diseases was discussed by Jordan [1] in 1999 to understand the dynamic interaction of the epidemic in a society and try to control it. The prevalence rate is expressed in a linear system of equations, which determines the development of the disease and presented as:

$$
\frac{d p}{d t}=-\beta p q
$$




$$
\begin{gathered}
\frac{d q}{d t}=\beta p q-\gamma q \\
\frac{d r}{d t}=\gamma q
\end{gathered}
$$

where $p(t)$ is the population at risk of infection but not yet infected, $q(t)$ is the affected and non-isolated population, and $r(t)$ is the isolated or recovered population. Using many analytical and semi-analytical methods, the system was one of the most widely solved by a group of researchers, including Biaza r[2], Rafei [3], Batiha [4], Dogan [5], and Weli [6]. At the present time, some researchers combined iterative methods with one of the known transformations, such as Laplace Adomian decomposition method [7], homotopy perturbation transform method [8], Aboodh decomposition method [9], and Temimi and Ansari method with the Elzaki transformation [10]. The coupling of TAM and LT leads to an effective and fast method compared to the other methods. In this method, no perturbation or liberalization is desired and it is easily implemented because it does not require complicated calculations of non-linear terms, as in the Adomian method.

\section{Algorithm of the new method (LTAM)}

To clarify ideas of the proposed LTAM method, let us consider the following nonlinear ordinary differential equation

with the initial condition

$$
L(w(t))=N(w(t))+g(t)
$$

$$
w(0)=f(t)
$$

where $\mathrm{L}$ is linear operator, $\mathrm{N}$ is a nonlinear operator, and $\mathrm{g}$ is a known function.

By taking LT on both sides of Eq. (2) (where we referred to the Laplace transform with the symbol $\wp$ ), we obtain

$$
\wp[L(w(t))]=\wp[N(w)(t)]+\wp[g(t)],
$$

Using the differentiation property of the LT, we obtain

$$
\begin{aligned}
& s(w(s))-w(0)=\wp[N(w)(t)]+\wp[g(t)], \\
& w(s)=\frac{f(t)}{s}+\frac{1}{s} \wp[N(w)(t)]+\frac{1}{s} \wp[g(t)],
\end{aligned}
$$

By taking the inverse of LT on both sides of Eq. (6), we obtain

$$
w(t)=\wp^{-1}\left[\frac{f(t)}{s}\right]+\wp^{-1}\left[\frac{1}{s} \wp[N(w)(t)]\right]+\wp^{-1}\left[\frac{1}{s} \wp[g(t)]\right],
$$

According to the TAM, the solution of Eq. (2) can be found by a simple iterative procedure. At first, the TAM method is used to find the initial approximation, which can be achieved by solving

Then, the next iteration will be

$$
w_{0}(t)=\wp^{-1}\left[\frac{f(t)}{s}\right]+\wp^{-1}\left[\frac{1}{s} \wp[g(t)]\right],
$$

$$
w_{1}(t)=w_{0}(t)+\wp^{-1}\left[\frac{1}{s} \wp\left[N\left(w_{0}\right)(t)\right]\right],
$$

After many iterations, the general form of the method is represented as

\section{The LTAM for Solving the Epidemic Model}

$$
w_{n+1}(t)=w_{0}(t)+\wp^{-1}\left[\frac{1}{s} \wp\left[N\left(w_{n}\right)(t)\right]\right],
$$

In this section, the epidemic model (1) with initial conditions

$p(0)=N_{1}, q(0)=N_{2}, r(0)=N_{3}$

will be solved by LTAM method.

By applying LT on both sides of the system of Eq.s (1), we obtain

$\wp\left[\frac{d p}{d t}\right]=\wp[-\beta p q]$

$$
\wp\left[\frac{d q}{d t}\right]=\wp[\beta p q]-\wp[\gamma q]
$$


$\wp\left[\frac{d r}{d t}\right]=\wp[\gamma q]$,

By using the differentiation property of the LT, we get $s p(s)-N_{1}=\wp[-\beta p q]$,

Hence, we simply get

$$
\begin{gathered}
s q(s)-N_{2}=\wp[\beta p q]-\wp[\gamma q], \\
\operatorname{sr}(s)-N_{3}=\wp[\gamma q]
\end{gathered}
$$

$p(s)=\frac{1}{s} N_{1}+\frac{1}{s} \wp[-\beta p q]$,

By taking $\mathrm{LT}$ as inverse, we have

$$
\begin{gathered}
q(s)=\frac{1}{s} N_{2}+\frac{1}{s} \wp[\beta p q]-\frac{1}{s} \wp[\gamma q], \\
r(s)=\frac{1}{s} N_{3}+\frac{1}{s} \wp[\gamma q]
\end{gathered}
$$

$$
\begin{gathered}
p(t)=\wp^{-1}\left[\frac{1}{s} N_{1}\right]+\wp^{-1}\left[\frac{1}{s} \wp[-\beta p q]\right], \\
q(t)=\wp^{-1}\left[\frac{1}{s} N_{2}\right]+\wp^{-1}\left[\frac{1}{s} \wp[\beta p q]\right]-\wp^{-1}\left[\frac{1}{s} \wp[\gamma q]\right], \\
r(t)=\wp^{-1}\left[\frac{1}{s} N_{3}\right]+\wp^{-1}\left[\frac{1}{s} \wp[\gamma q]\right]
\end{gathered}
$$

According to the TAM, the solution of the system of Eq.s (1) can be found by simple a iterative procedure. Firstly, we find the initial approximation and this can be achieved by solving the following:

$$
\begin{aligned}
& p_{0}(t)=\wp^{-1}\left[\frac{1}{s} N_{1}\right]=N_{1} \\
& q_{0}(t)=\wp^{-1}\left[\frac{1}{s} N_{2}\right]=N_{2}, \\
& r_{0}(t)=\wp^{-1}\left[\frac{1}{s} N_{3}\right]=N_{3}
\end{aligned}
$$

The second approximation can be found by solving

$$
\begin{gathered}
p_{1}(t)=\wp^{-1}\left[\frac{1}{s} N_{1}\right]+\wp^{-1}\left[\frac{1}{s} \wp\left[-\beta p_{0} q_{0}\right]\right]=-N_{1} N_{2} t \beta \\
q_{1}(t)=\wp^{-1}\left[\frac{1}{s} N_{2}\right]+\wp^{-1}\left[\frac{1}{s} \wp\left[\beta p_{0} q_{0}\right]\right]-\wp^{-1}\left[\frac{1}{s} \wp\left[\gamma q_{0}\right]\right]=N_{1} N_{2} t \beta-N_{2} t \gamma \\
r_{1}(t)=\wp^{-1}\left[\frac{1}{s} N_{3}\right]+\wp^{-1}\left[\frac{1}{s} \wp\left[\gamma q_{0}\right]\right]=N_{2} t \gamma
\end{gathered}
$$

By the same way, we find the rest of the third, fourth, and fifth approximations

$$
\begin{gathered}
p_{2}(t)=\wp^{-1}\left[\frac{1}{s} N_{1}\right]+\wp^{-1}\left[\frac{1}{s} \wp\left[-\beta p_{1} q_{1}\right]\right]=\frac{1}{3} N_{1} N_{2} \beta^{2}\left(N_{1} N_{2} t^{3} \beta-N_{2} t^{3} \gamma\right) \\
q_{2}(t)=\wp^{-1}\left[\frac{1}{s} N_{2}\right]+\wp^{-1}\left[\frac{1}{s} \wp\left[\beta p_{1} q_{1}\right]\right]-\wp^{-1}\left[\frac{1}{s} \wp\left[\gamma q_{1}\right]\right]=-\frac{1}{2} \gamma\left(N_{1} N_{2} t^{2} \beta-N_{2} t^{2} \gamma\right)- \\
\frac{1}{3} N_{1} N_{2} \beta^{2}\left(N_{1} N_{2} t^{3} \beta-N_{2} t^{3} \gamma\right) \\
r_{2}(t)=\wp^{-1}\left[\frac{1}{s} N_{3}\right]+\wp^{-1}\left[\frac{1}{s} \wp\left[\gamma q_{1}\right]\right]=\frac{1}{2} \gamma\left(N_{1} N_{2} t^{2} \beta-N_{2} t^{2} \gamma\right) \\
p_{3}(t)=\wp^{-1}\left[\frac{1}{s} N_{1}\right]+\wp^{-1}\left[\frac{1}{s} \wp\left[-\beta p_{2} q_{2}\right]\right]=\frac{1}{252} N_{1} N_{2}^{3} t^{6} \beta^{3}\left(-N_{1} \beta+\gamma\right)^{2}\left(4 N_{1} N_{2} t \beta^{2}+7 \gamma\right) \\
q_{3}(t)=\wp^{-1}\left[\frac{1}{s} N_{2}\right]+\wp^{-1}\left[\frac{1}{s} \wp\left[\beta p_{2} q_{2}\right]\right]-\wp^{-1}\left[\frac{1}{s} \wp\left[\gamma q_{2}\right]\right] \\
=-\frac{1}{252} N_{2} t^{3}\left(N_{1} \beta-\gamma\right)\left(4 N_{1}{ }^{3} N_{2}{ }^{3} t^{4} \beta^{6}+N_{1}{ }^{2} N_{2}{ }^{2} t^{3} \beta^{4}\left(7-4 N_{2} t \beta\right) \gamma-42 \gamma^{2}\right. \\
\left.-7 N_{1} N_{2} t \beta^{2} \gamma\left(3+N_{2} t^{2} \beta \gamma\right)\right)
\end{gathered}
$$




$$
\begin{aligned}
& r_{3}(t)=\wp^{-1}\left[\frac{1}{s} N_{3}\right]+\wp^{-1}\left[\frac{1}{s} \wp\left[\gamma q_{2}\right]\right]=-\frac{1}{12} N_{2} t^{3}\left(N_{1} \beta-\gamma\right) \gamma\left(N_{1} N_{2} t \beta^{2}+2 \gamma\right) \\
& p_{4}(t)= \\
& \wp^{-1}\left[\frac{1}{s} N_{1}\right]+\wp^{-1}\left[\frac{1}{s} \wp\left[-\beta p_{3} q_{3}\right]\right]=\frac{1}{136216080} N_{1} N_{2}{ }^{4} t^{10} \beta^{4}\left(N_{1} \beta-\gamma\right)^{3}\left(2288 N_{1}{ }^{4} N_{2}{ }^{4} t^{5} \beta^{8}-\right. \\
& 572 N_{1}{ }^{3} N_{2}{ }^{3} t^{4} \beta^{6}\left(-15+4 N_{2} t \beta\right) \gamma-63063 \gamma^{3}-165 N_{1}{ }^{2} N_{2}{ }^{2} t^{2} \beta^{4} \gamma(91-49 t \gamma+ \\
& \left.\left.52 N_{1}{ }_{2} t^{2} \beta \gamma\right)-105 N_{1} N_{2} t \beta^{2} \gamma^{2}\left(585+77 N_{2} t^{2} \beta \gamma\right)\right) \\
& q_{4}(t)=\wp^{-1}\left[\frac{1}{s} N_{2}\right]+\wp^{-1}\left[\frac{1}{s} \wp\left[\beta p_{3} q_{3}\right]\right]-\wp^{-1}\left[\frac{1}{s} \wp\left[\gamma q_{3}\right]\right]= \\
& \frac{1}{2520} N_{2} t^{4}\left(N_{1} \beta-\gamma\right) \gamma\left(5 N_{1}{ }^{3} N_{2}{ }^{3} t^{4} \beta^{6}-5 N_{1}{ }^{2} N_{2}{ }^{2} t^{3} \beta^{4}\left(-2+N_{2} t \beta\right) \gamma-105 \gamma^{2}-\right. \\
& \left.2 N_{1} N_{2} t \beta^{2} \gamma\left(21+5 N_{2} t^{2} \beta \gamma\right)\right)-\frac{1}{136216080} N_{1} N_{2}{ }^{4} t^{10} \beta^{4}\left(N_{1} \beta-\gamma\right)^{3}\left(2288 n 1^{4} n 2^{4} t^{5} \beta^{8}-\right. \\
& 572 N_{1}{ }^{3} N_{2}{ }^{3} t^{4} \beta^{6}\left(-15+4 N_{2} t \beta\right) \gamma-63063 \gamma^{3}-165 N_{1}{ }^{2} N_{2}{ }^{2} t^{2} \beta^{4} \gamma(91-49 t \gamma+ \\
& \left.\left.52 N_{2} t^{2} \beta \gamma\right)-105 N_{1} N_{2} t \beta^{2} \gamma^{2}\left(585+77 N_{2} t^{2} \beta \gamma\right)\right) \\
& r_{4}(t)=\wp^{-1}\left[\frac{1}{s} N_{3}\right]+\wp^{-1}\left[\frac{1}{s} \wp\left[\gamma q_{3}\right]\right]=-\frac{1}{2520} N_{2} t^{4}\left(N_{1} \beta-\gamma\right) \gamma\left(5 N_{1}{ }^{3} N_{2}{ }^{3} t^{4} \beta^{6}-\right. \\
& \left.5 N_{1}{ }^{2} N_{2}{ }^{2} t^{3} \beta^{4}\left(-2+N_{2} t \beta\right) \gamma-105 \gamma^{2}-2 N_{1} N_{2} t \beta^{2} \gamma\left(21+5 N_{2} t^{2} \beta \gamma\right)\right)
\end{aligned}
$$

To confirm the accuracy of the solution, we compared the solutions obtained by using LTAM for 5-term approximation with the numerical solutions calculated using MATHEMATICA software. Moreover, we proposed the following error remainder functions for the epidemic model to verify the accuracy of the results:

$$
\begin{aligned}
& \mathrm{ER}_{p_{n}}=\frac{d}{d t}\left(\sum_{i=0}^{n}(p i)+\beta\left[\left(\sum_{i=0}^{n}(p i)+\sum_{i=0}^{n}(q i)\right]=0,\right.\right. \\
& \mathrm{ER}_{\mathrm{q}_{\mathrm{n}}}=\frac{d}{d t}\left(\sum_{i=0}^{n}(q i)-\beta\left[\left(\sum_{i=0}^{n}(p i) * \sum_{i=0}^{n}(q i)\right]+\gamma\left(\sum_{i=0}^{n}(q i)\right]=0,\right.\right. \\
& \mathrm{ER}_{\mathrm{r}_{\mathrm{n}}}=\frac{d}{d t}\left(\sum_{i=0}^{n}(r i)-\gamma\left(\sum_{i=0}^{n}(q i)\right]=0,\right.
\end{aligned}
$$

with the maximal error remainder parameter respectively ,

$$
\begin{aligned}
\operatorname{MERp}_{\mathrm{n}}= & \max \left|\operatorname{ERp}_{\mathrm{n}}(\mathrm{t})\right| \\
& 0 \leq \mathrm{x} \leq 1 \\
\operatorname{MERq}_{\mathrm{n}}= & \max \left|\operatorname{ERq}_{\mathrm{n}}(\mathrm{t})\right| \\
& 0 \leq \mathrm{x} \leq 1 \\
\operatorname{MERr}_{\mathrm{n}}= & \max \left|\operatorname{ERr}_{\mathrm{n}}(\mathrm{t})\right| \\
& 0 \leq \mathrm{x} \leq 1
\end{aligned}
$$

\section{Numerical Comparison and Simulation}

We take the parameter values (see [2]), as follows:

$N_{1}=20=$ Initial population of $p(t)$ who are susceptible.

$N_{2}=15=$ Initial population of $q(t)$ who are infective.

$N_{3}=10=$ Initial population of $r(t)$ who are immune.

$\beta=0.01=$ Rate of change of susceptible population to infective population.

$\gamma=0.02=$ Rate of change of infective population to immune population.

The values of the coefficients above will be offset in Eq.s (16), (17), (18), and (19). Then, we get the following results:

$$
\begin{gathered}
p_{1}=20-3 t \\
q_{1}=15+2.7 t \\
r_{1}=10+0.3 t
\end{gathered}
$$




$$
\begin{aligned}
& \quad q_{3}=15+2.7 t+0.018 t^{2}-0.02817 t^{3}-0.00064125 t^{4}+0.00030618 t^{5}+2.835 \times \\
& \quad 10^{-6} t^{6}-1.04143 \times 10^{-6} t^{7} \\
& r_{3}=10+0.3 t+0.027 t^{2}+0.00012 t^{3}-0.000135 t^{4} \\
& p_{4}=20-3 . t-0.045 t^{2}+0.02805 t^{3}+0.000795375 t^{4}-0.000316508 t^{5}- \\
& 0.0000122051 t^{6}+3.54056 \times 10^{-6} t^{7}+1.00638 \times 10^{-7} t^{8}-2.49702301607143 \times \\
& 10^{-8} t^{9}-6.59004 \times 10^{-10} t^{10}+1.34797 \times 10^{-10} t^{11}+2.67689 \times 10^{-12} t^{12}-4.84378 \times \\
& 10^{-13} t^{13}-4.21779 \times 10^{-15} t^{14}+7.23049 \times 10^{-16} t^{15} \\
& q_{4}=15+2.7 t+0.018 t^{2}-0.02817 t^{3}-0.000654525 t^{4}+0.000319073 t^{5}+ \\
& 0.0000111845 t^{6}-3.54866 \times 10^{-6} t^{7}-9.80339 \times 10^{-8} t^{8}+2.49702 \times 10^{-8} t^{9}+ \\
& 6.59004 \times 10^{-10} t^{10}-1.34797 \times 10^{-10} t^{11}-2.67689 \times 10^{-12} t^{12}+4.84378 \times \\
& 10^{-13} t^{13}+4.21779 \times 10^{-15} t^{14}-7.23049 \times 10^{-16} t^{15} \\
& r_{4}=10+0.3 t+0.027 t^{2}+0.00012 t^{3}-0.00014085 t^{4}-2.565 \times 10^{-16} t^{5}+1.0206 \times \\
& 10^{-16} t^{6}+8.1 \times 10^{-9} t^{7}-2.60357 \times 10^{-9} t^{8}
\end{aligned}
$$

Figures 1, 2, and 3 illustrate the graphs of the results. The plots show the size of the population, which was constant during the period of the epidemic. The number of people infected with the infection increases but the number of people exposed to infection decreases, while we notice an increase in the number of immune people. When comparing the results obtained by using the LTAM method with the semi-analytical methods in previous studies, we note that the results are identical to the fifth term with VIM [3], DTM [4], and DJM [6], and identical to the sixth term with ADM [2].

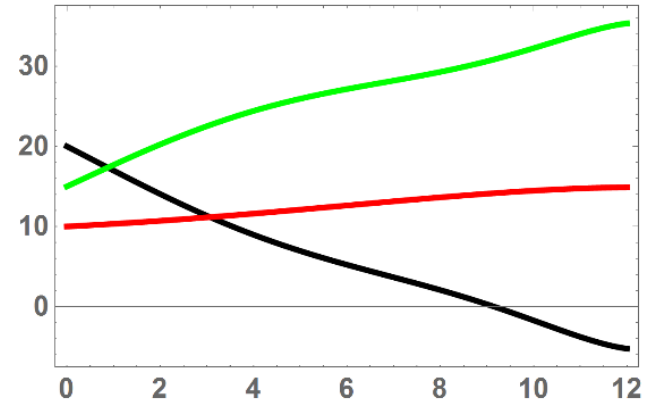

Figure 1-Three terms approximation for $\mathrm{p}(\mathrm{t}), \mathrm{q}(\mathrm{t}), \mathrm{r}(\mathrm{t})$

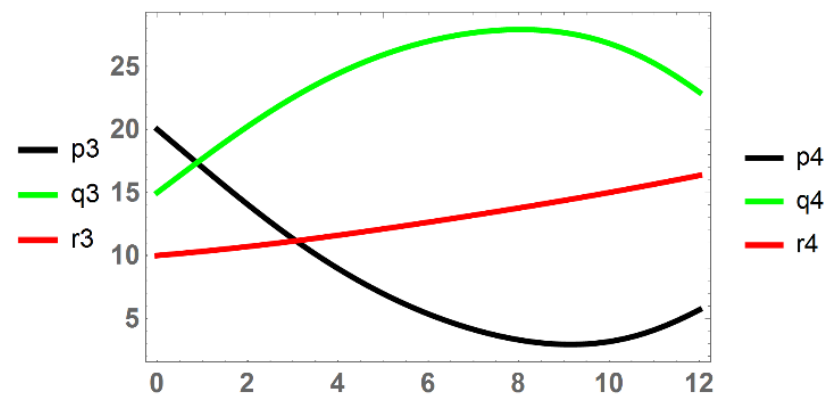

Figure 2- Four terms approximation for $\mathrm{p}(\mathrm{t}), \mathrm{q}(\mathrm{t}), \mathrm{r}(\mathrm{t})$

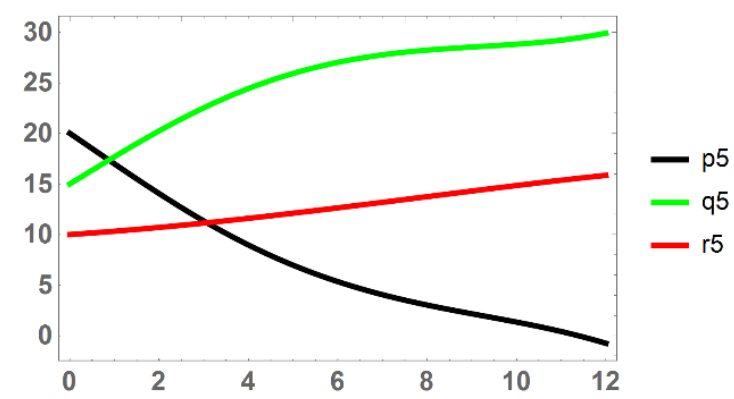

Figure 3- Five terms approximation for $\mathrm{p}(\mathrm{t}), \mathrm{q}(\mathrm{t}), \mathrm{r}(\mathrm{t})$. 


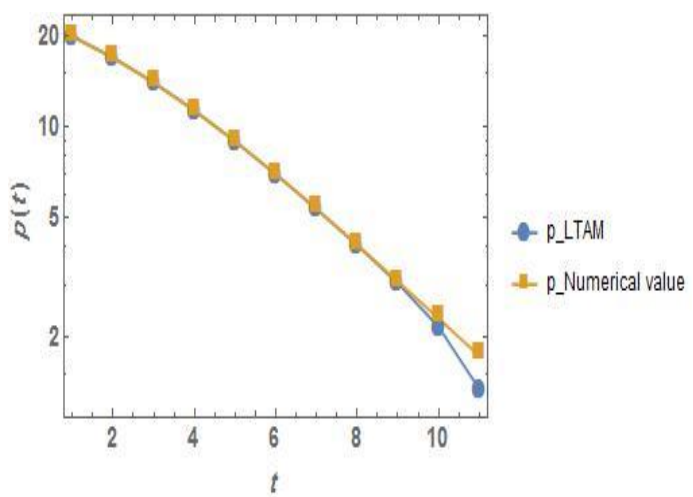

Figure 4 Comparison between the curves of of the approximate $\mathrm{p}(\mathrm{t})$ by using LTAM and the numerical function for $0 \leq \mathrm{t} \leq 10$

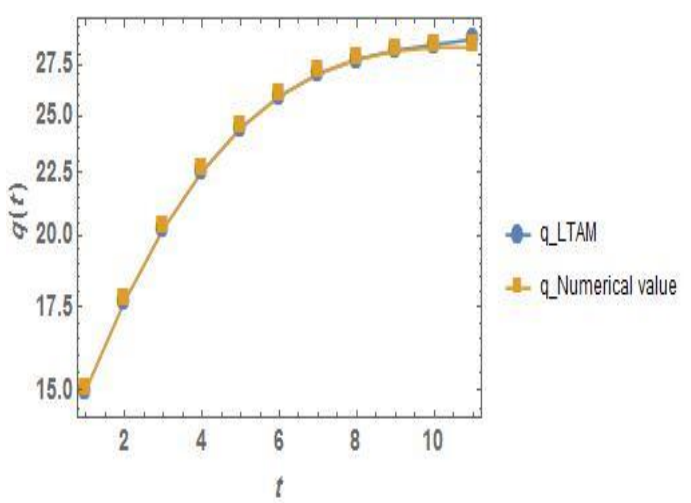

Figure 5- Comparison between the curves the approximate $\mathrm{q}(\mathrm{t})$ by using LTAM and the numerical function for $0 \leq \mathrm{t} \leq 10$

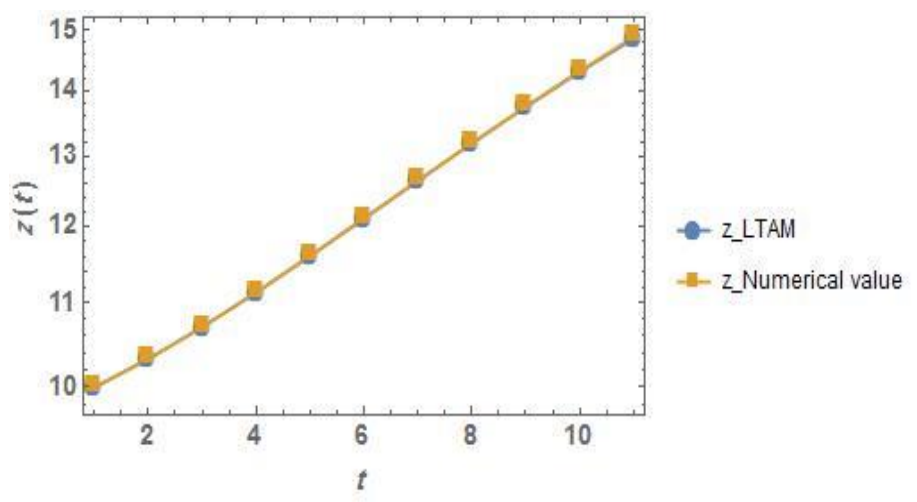

Figure 6- Comparison between the curves of the approximate $\mathrm{z}(\mathrm{t})$ by using LTAM and the numerical function for $0 \leq \mathrm{t} \leq 10$

To indicate the validation of the LTAM, Figures 4, 5, and 6 present the difference between the approximate solutions, which is created by LTAM, and the numerical solution.

On other side, the absolute error resulting from the numerical method and the tenth iteration of the LTAM are calculated. We attain a good approximation, as shown in Table 1.

Table 1-Absolute errors obtained by using a numerical method and LTAM for ten approximations

\begin{tabular}{|c|c|c|c|}
\hline $\mathrm{t}_{\mathrm{i}}$ & $\mathrm{p}_{\mathrm{i}}-\mathrm{N}_{\mathrm{p}}$ & $\mathrm{q}_{\mathrm{i}}-\mathrm{N}_{\mathrm{q}}$ & $\mathrm{z}_{\mathrm{i}}-\mathrm{N}_{\mathrm{z}}$ \\
\hline 0 & 0 & 0 & 0 \\
\hline 1 & $3.58804801 \times 10^{-7}$ & $9.635815346 \times 10^{-8}$ & $2.624466475 \times 10^{-7}$ \\
\hline 2 & $3.097099466 \times 10^{-9}$ & $2.579191651 \times 10^{-7}$ & $2.548220639 \times 10^{-7}$ \\
\hline 3 & $8.032454861 \times 10^{-7}$ & $1.06397777 \times 10^{-6}$ & $2.607322891 \times 10^{-7}$ \\
\hline 4 & $1.558973164 \times 10^{-7}$ & $7.719740225 \times 10^{-8}$ & $2.330947204 \times 10^{-7}$ \\
\hline 5 & $2.949319073 \times 10^{-7}$ & $6.484871662 \times 10^{-8}$ & $2.300831881 \times 10^{-7}$ \\
\hline 6 & $7.831758841 \times 10^{-7}$ & $9.614238294 \times 10^{-7}$ & $1.782479373 \times 10^{-7}$ \\
\hline 7 & $9.059311878 \times 10^{-8}$ & $2.925829783 \times 10^{-7}$ & $2.019898506 \times 10^{-7}$ \\
\hline 8 & $6.028532908 \times 10^{-7}$ & $4.923535428 \times 10^{-7}$ & $1.104997427 \times 10^{-7}$ \\
\hline 9 & $7.934803869 \times 10^{-6}$ & $8.412187384 \times 10^{-6}$ & $4.773835194 \times 10^{-7}$ \\
\hline 10 & $6.924430771 \times 10^{-5}$ & $7.47748496 \times 10^{-5}$ & $5.530541889 \times 10^{-6}$ \\
\hline
\end{tabular}




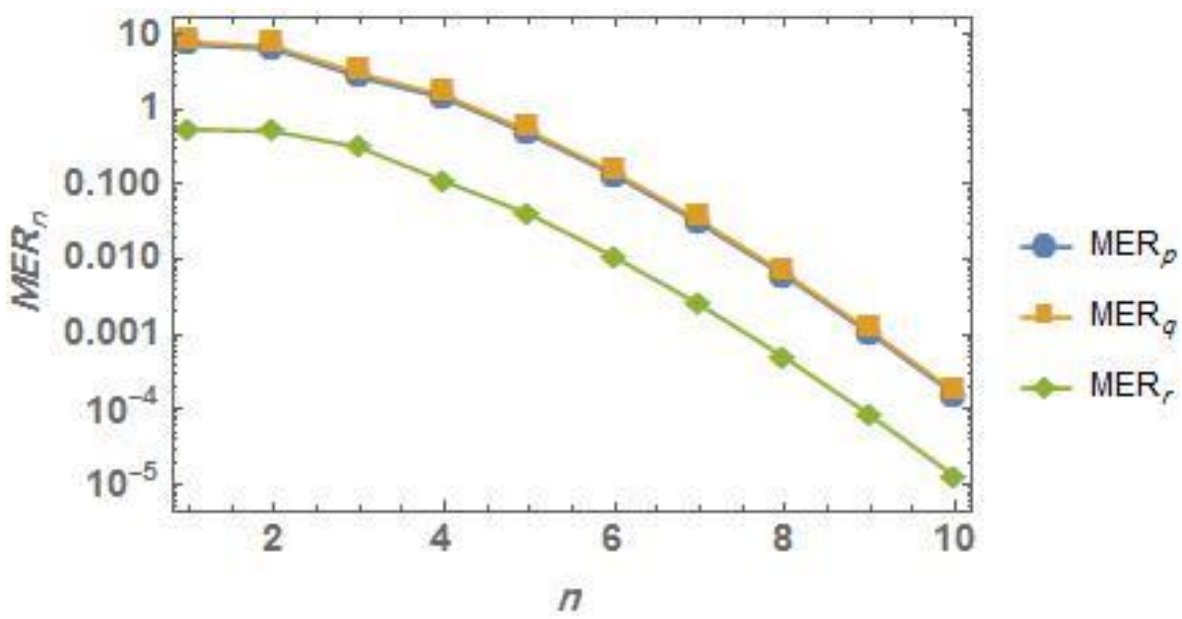

Figure 7- The maximum error remainder plots for the epidemic model problem by using the LTAM

The logarithmic sketch of the maximum error remainder parameters $\mathrm{MER}_{n}$ for $n=1$ to 10 is explained in Figure 7, where an exponential rate of convergence can be noticed.

Table 2-The residual errors obtained by LTAM solutions

\begin{tabular}{|c|c|c|c|}
\hline $\mathrm{t}$ & Residual error $_{\mathrm{p}}$ & Residual error ${ }_{q}$ & Residual error $_{\mathrm{r}}$ \\
\hline 0 & 0 & 0 & 0 \\
\hline 1 & $2.9792310^{-9}$ & $7.76305 \times 10^{-9}$ & $4.78382 \times 10^{-9}$ \\
\hline 2 & $5.29561 \times 10^{-7}$ & $5.40947 \times 10^{-7}$ & $1.13858 \times 10^{-8}$ \\
\hline 3 & $4.50258 \times 10^{-6}$ & $6.44414 \times 10^{-6}$ & $1.94156 \times 10^{-6}$ \\
\hline 4 & $6.42312 \times 10^{-5}$ & $7.56359 \times 10^{-5}$ & $1.14047 \times 10^{-5}$ \\
\hline 5 & $3.68225 \times 10^{-5}$ & $4.10924 \times 10^{-5}$ & $4.26993 \times 10^{-6}$ \\
\hline 6 & $2.72889 \times 10^{-3}$ & $3.01795 \times 10^{-3}$ & $2.89061 \times 10^{-4}$ \\
\hline 7 & $1.75119 \times 10^{-2}$ & $1.91747 \times 10^{-2}$ & $1.66274 \times 10^{-3}$ \\
\hline 8 & $6.79616 \times 10^{-2}$ & $7.39966 \times 10^{-2}$ & $6.03504 \times 10^{-3}$ \\
\hline 9 & $1.96927 \times 10^{-1}$ & $2.13825 \times 10^{-1}$ & $1.68975 \times 10^{-2}$ \\
\hline 10 & $4.62112 \times 10^{-1}$ & $5.01524 \times 10^{-1}$ & $3.94118 \times 10^{-2}$ \\
\hline
\end{tabular}

Finally, if we compare LTAM with other numerical methods, we find that LTAM do not need to use any kind of truncation errors to estimate the accuracy of the approximate solution. There is also no need for selecting the step size of the subintervals over the whole interval in the LTAM. Additionally, we do not need to create any round-off errors.

\section{Conclusions}

In this study, an iterative method is offered to solve the nonlinear system of differential equations that control the problem of an epidemic model. This method is more featured and effective because it provides the analytical solution straightly without any discretion or linearity. According to the figures, it has to be noted that the maximum error remainders are decreased when the number of iterations is increased.

Numerical results of the TAM are measured with those of the numerical value calculated by using the MATHEMATICA program.

When comparing the solution obtained by LTAM with those offered by some other methods, such as ADM, HPM, HAM, and VIM, it is appreciated that the approximate solutions acquired by the LTAM converge speedly without any restricted hypotheses. Thus, LTAM seems to ensure very reliable results with high accuracy. 


\section{Reference}

[1] D. W. Jordan and P. Smith, Nonlinear ordinary differential equations, third ed., Oxford University Press, 1999, p. 64 .

[2] J. Biazar, "Solution of the epidemic model by Adomian decomposition method," Applied Mathematics and Computation, vol. 173, no. 2, pp. 1101-1106, 2006.

[3] M. Rafei, H. Daniali and D.D. Ganji, "Variational iteration method for solving the epidemic model and the prey and predator problem," Applied Mathematics and Computation, vol. 186, no. 2, pp. 1701-1709, 2007.

[4] A. M. Batiha and B. Batiha, "A new method for solving epidemic model," Australian Journal of Basic and Applied Sciences, vol. 5, no. 12, pp. 3122-3126, 2011.

[5] N. Dogan and O.Akin, "Series solution of Epidemic model," TWMS Journal of Applied and Engineering Mathematics, vol. 2, no. 2, pp. 238-244, 2012.

[6] M. A. Weli, "A reliable iterative method for solving the epidemic model and the prey and predator problems," International Journal of Basic \& Applied Sciences, vol. 3, no.4, pp. 441-450, 2014.

[7] Sh. Sh. Ahmed, Sh. A. H. Salih, and M. R. Ahmed, "Laplace Adomian and Laplace modified Adomian decomposition methods for solving nonlinear Integro-Fractional differential equations of the Volterra-Hammerstein type," Iraqi Journal of Science, vol. 60, no. 10, pp. 2207-2222, 2019.

[8] M. Suleman, Q. Wu and Gh. Abbas, "Approximate analytic solution of $(2+1)$ dimensional coupled differential Burger's equation using Elzaki Homotopy Perturbation Method," Alexandria Engineering Journal, vol. 55, no. 2, pp. 1817-1826, 2016.

[9] R. I. Nuruddeen, and A. M. Nass, "Aboodh decomposition method and its application in solving linear and nonlinear heat equations," European Journal of Advances in Engineering and Technology, vol. 3, no, 7, pp. 34-37, 2016.

[10]A.S. Mohammed, "The new combination of semi-analytical iterative method and Elzaki transform for solving some Korteweg-de Vries equations," Al-Qadisiyah Journal Of Pure Science, vol. 25, no. 1, pp. $23-26,2020$. 